\title{
Shaping the HIV epidemic in Poland - proceedings from the first Polish workshop on cascade of care in HIV
}

Justyna D. Kowalska ${ }^{1,2}$, Anna Grzeszczuk ${ }^{3}$, Karolina Pyziak-Kowalska ${ }^{1,2}$, Anna Marzec-Bogusławska ${ }^{4}$, Magdalena Rosińska ${ }^{5}$, Magdalena Ankiersztejn-Bartczak ${ }^{6}$, Andrzej Horban ${ }^{1,2}$

${ }^{1}$ Medical University of Warsaw, Poland

${ }^{2}$ Hospital for Infectious Diseases in Warsaw, Poland

${ }^{3}$ Medical University of Bialystok, Poland

${ }^{4}$ National AIDS Centre, Ministry of Health, Poland

${ }^{5}$ National Institute of Public Health, Poland

${ }^{6}$ Foundation of Social Education, Poland

\begin{abstract}
In October 2016, a workshop was held in Warsaw gathering experts and activists involved in different areas of human immunodeficiency virus (HIV) response. The purpose of this meeting was to reassess current needs in controlling the HIV epidemic in Poland in relation to recently launched UNAIDS goal of 90-90-90. During the meeting, several points of action were established to be considered as key factors to improve the response to HIV epidemic in Poland. Those were estimating the number of people living with HIV in Poland using mathematical modelling based on real data sampling, expanding new testing options in non-medical settings, i.e. rapid testing, co-testing (HIV, HCV, STI), strengthening linkage to care after establishing the HIV diagnosis in non-medical settings, expanding HIV testing in medical settings, starting with introducing routine HIV testing for indicator conditions as a standard procedure across different medical specialties, knowing the number of HIV persons already in care, and on effective cART, continuing to fight stigma and discrimination through the means of social education and counselling.
\end{abstract}

HIV AIDS Rev 2017; 16, 2: 130-133 DOI: https://doi.org/10.5114/hivar.2017.68804

Key words: HIV, Poland, cascade of care, testing, antiretroviral therapy.

\section{Introduction}

Early diagnosis and initiation of combination antiretroviral therapy (cART) reduce morbidity and mortality of human immunodeficiency virus (HIV)-positive people to a significant degree $[1,2]$. It also affects the rates of onward HIV transmission and health care costs related to medical care of new HIV infections [3, 4]. All above can be only as- sumed if patients, who are aware of their HIV status, are also linked to care and remain under effective cART [5].

Early cART initiation has been linked to better prognosis, in terms of preventing both AIDS and non-AIDS events $[6,7]$. However, data presented from a long-term pan-European study show significantly higher proportion of AIDSrelated mortality in the eastern European region, indicating clear inequalities in standards of care across Europe $[8,9]$.
Address for correspondence: Dr Justyna Dominika Kowalska, Department of Adults' Infectious Diseases, Medical University of Warsaw, 37 Wolska St., 01-201 Warsaw, Poland,

e-mail: jdkowalska@gmail.com
Article history:

Received: 29.01.2017

Received in revised form: 24.03.2017

Accepted: 19.05.2017

Available online: 05.07 .2017
International Journal of HIV-Related Problems

HIV \& AIDS

R e vi e w 
A 90-90-90 strategy presented by UNAIDS addresses the key elements of successful HIV care programs, setting the goals that $90 \%$ of HIV infected should be diagnosed, of those $90 \%$ should be treated, and of those treated, 90\% should be virally suppressed. Achievement of these aims requires that countries know their HIV epidemic in order to better target the interventions [10,11]. National data on cascades of care in central and eastern Europe are sparse, but the work published shows low proportions of people linked to care and on cART $[12,13]$. On the other hand, high rates of suppression are achieved among patients in care and on CART in some countries, showing that more emphasis needs to be put on HIV testing and linking those diagnosed to care [14-16].

In October 2016, a workshop was held in Warsaw gathering experts and activists involved in different areas of response to the HIV epidemic. The purpose of this meeting was to reassess current needs in controlling the HIV epidemic in Poland in relation to the recently launched UNAIDS goal of 90-90-90. The workshop was sponsored by Optimizing Testing and Linkage to Care across Europe (OptTEST) project, which is co-funded by $2^{\text {nd }}$ Health Programme of the European Union.

\section{Meeting proceedings}

\section{Overview of European activities in the field}

During the first day of the meeting, the invited speakers presented current European activities in the field of monitoring of the continuum of HIV care underlining solutions to specific problems faced by other European countries. Stine Finne Jakobsen (CHIP, Centre of Excellence for Health, Immunity and Infections, Copenhagen, Denmark) introduced an overview of the OptTEST project, which focuses on reducing the number of undiagnosed people with HIV infection, as well as promoting timely treatment and care delivery. Other European initiatives being co-financed by the European Commission's Health Programmes are Euro HIV Edat, HA-React, SIALON II, ESTICOM, and Link2Care. Caroline Rae (Saint Stephen's AIDS Trust, London, United Kingdom) presented experiences from the study 'HIV indicator diseases across Europe' (HIDES) run by the HIV in Europe initiative [17]. The HIDES 1 study assessed HIV prevalence in eight indicators of the disease in specific populations. Several countries from Europe, including Poland, took part in HIDES 1 and 2 study, providing a proof on concept from real-life clinical settings [18]. Jordi Casabona (Euro HIV EDAT, Center of Epidemiological Studies on HIV and STI, Catalonia) demonstrated data from monitoring community-based voluntary counselling and testing centers (CBVCTs), which were a part of COBATEST network. This European initiative included 55 CBVCTs from 22 different countries in a questionnaire based study. The study revealed that the majority of CBVCTs in Europe are predominantly fo- cused on men who have sex with men (MSM), and CBVCTs targeting other at-risk populations were more likely to be medicalized. The COBATEST underlined the necessity for sustaining the CBVCTs heterogeneity, both in accessing low threshold risk groups and in the form of testing, yet emphasizing the development of standardized indicators to enable comparisons over time, aiming for improvement the effectiveness of CBVCTs across Europe [19]. Improvement of testing in medical settings was also discussed, striking the need of individualized and informed approach to patients. Valerie Delpech (Public Health England, London, United Kingdom) presented possible benefits coming out of monitoring of the HIV epidemic, and of the quality of care basing on experience from building the monitoring system in the United Kingdom. This system uses a non-reversible, personalized identification code providing both precision for surveillance (i.e., allowing de-duplication and linkage of data-sources), and confidentiality for an individual person. Accurate measurements allowed to monitor and interact with real life situation. In result in 2014, in the United Kingdom, all subpopulations of people living with HIV reached the UNAIDS targets of $90 \%$ diagnosed on cART and $90 \%$ with viral load suppression for those on cART. There is no indication of inequalities in HIV care received through the National Health Service by gender, ethnicity, and HIV exposure. The monitoring system was also able to capture young people living with diagnosed HIV having suboptimal outcomes.

Kamilla Groenborg Laut (CHIP, Centre of Excellence for Health, Immunity and Infections, Copenhagen, Denmark) showed a new methodology applied to investigating the right side of the cascade of care in the EuroSIDA study. Briefly, EuroSIDA is a prospective observational cohort study with 100 collaborating clinics in 35 European countries plus Israel and Argentina, and includes around 15,000 HIV-positive people under active follow-up [20]. Of note, only six out of the 35 countries participating in EuroSIDA reached levels of ART-coverage and virological suppression among those on ART that were above $90 \%$. These analyses also showed that HIV-positive patients from eastern Europe were $80 \%$ less likely to be virologically suppressed, while on cART as compared to patients from western Europe [21]. This underlines the need for reassessment of both treatment access and its effectiveness.

Finally, Lara Tavoschi (The European Centre for Disease Prevention and Control, ECDC, Stockholm, Sweden) discussed the perspectives for joining the monitoring of the two edges of cascade of care in Europe. She underlined that most of European countries require strengthening the collaboration between the national surveillance and the HIV care centers in order to better understand the current situation and, therefore, to properly target the interventions. ECDC's survey following the Dublin Declaration revealed that $78 \%$ of the responding countries from the WHO European region had significant gaps in their national continuum of care relating to timely HIV diagnosis, $41 \%$ of countries recognized difficulties in linking those diagnosed into care, and 
$48 \%$ of countries had break points in getting those in care on treatment $[22,23]$.

\section{Overview of the Polish HIV epidemic and key points from general discussion}

The second day of the workshop focused on the HIV epidemic in Poland, and whether there are data allowing informed and structured interventions, namely the cascade of care. The left side of cascade of care, which included testing and epidemiological surveillance, was presented by Anna Marzec-Bogusławska (National AIDS Centre, Ministry of Health, Poland), Magdalena Rosińska (National Institute of Public Health, Poland), Karolina Pyziak-Kowalska (Hospital for Infectious Diseases in Warsaw, Poland), and Anna Grzeszczuk (Medical University of Białystok, Poland). The right side of cascade of care, included linkage to care and antiretroviral treatment outcomes, was presented by Andrzej Horban (National Consultant in Infectious Diseases, Poland) and Magdalena Ankiersztejn-Bartczak (Foundation of Social Education, Poland).

Several key areas to improve the response of the HIV epidemic were identified by the speakers as well as during subsequent open discussion. The participants focused on identifying challenges and opportunities to meet the 90-9090 goal in Poland. First of all, it was strongly expressed that the existing HIV surveillance system requires amendments to better represent the current national epidemiology of HIV infection. It was established over two decades ago, and since then, the healthcare system and settings has changed very much. Current surveillance system served well to identify major trends in epidemic, such as shift from HIV transmission from injecting drug use to sexual contacts, or differences in the regional prevalence in HIV infection. However, in its existing form it is subject to double reporting error due to the legally introduced option of reporting without an unique identifier, as well as vulnerable towards incomplete data submission. For example for 5,615 (60.5\%) new HIV diagnoses registered between 2000 and 2011, the mode of HIV transmission was not reported [24].

Briefly, the system requires laboratory confirming HIV diagnosis and clinician diagnosing HIV to notify the case to a local Sanitary-Epidemiological Station (Stacja Sanitarno-Epidemiologiczna, Sanepid). The Sanepid registers the received notifications of cases residing in their jurisdiction, and forwards the other ones to the appropriate Sanepid. The local Sanepid integrates available reports on one case and forwards the information to regional Sanepid, and then centrally. De-duplication procedure is repeated at each level, as the patient's address may be miss-recorded or the patient could have moved. De-duplication is only possible if a unique identifier is used. In case of anonymized reports, the confirmatory test numbers and the date of the test are also used to identify possible duplicates. However, not all clinical reports can be linked to laboratory reports, leading to substantial level of missing information on the import- ant variables. Moreover, the current system does not allow for auditing and quality cross checks for the missing data or error inputs.

At the same time, we are facing large underestimation of the number of people living with HIV in Poland. Modelling analyses published by Rosińska et al. underlined that the difference between identified and estimated number of HIV-positive persons among MSM in Poland is substantial [25].

It has been mentioned that introducing an unique identifier for new cases is a necessity in order to improve the management of surveillance and to track the cascade of care.

It has been clearly shown that there is a need for expanding existing HIV testing both in non- and medical settings in Poland. A clear achievement in this field is a system of facility based counselling and testing centers (VCTs) developed in Poland, as part of National Programme for Combating AIDS and Preventing HIV Infections, and supervised by the National AIDS Centre Programme. Approximately, one in three HIV-positive persons in Poland is diagnosed through this system. The VCTs system is mainly targeting populations at risk and people with risk exposures/behaviors, such as men-having sex with men or people who have unprotected sex while using drugs or alcohol. However, a clear challenge is to expand HIV testing on populations unaware of the risks or in denial of such. As discussed during the meeting, new testing approaches, especially home based testing, could put surveillance system at a completely unknown land.

Another challenge is expanding testing in medical facilities. As presented during the meeting, indicator condition-based HIV testing in Polish settings is possible and proves to be effective in detecting new HIV infections [17, 26]. Although it is recommended by Polish AIDS Society, it is frequently not incorporated into other medical guidelines, thus not a standard of care [27]. A good example is mononucleosis-like illness that may be a clinical manifestation of early HIV infection, which occurs during the time of the patient's highest infectiousness. The positivity rate for routine HIV testing of patients presenting with mononucleosis-like illness in the Emergency Department of Hospital for Infectious Diseases in Warsaw was 5.5\% with linkage to care and starting antiretroviral therapy covering $100 \%$ of diagnosed patients [26]. Currently available data suggest that linkage to HIV care is lower for patients tested in non-medical settings, with people of hetero- or bisexual orientation, and lower education or knowledge about HIV being at higher risk of not being linked to care [28]. While linkage to care did not improve over time, the time to start the treatment and proportion of patients starting cART significantly improved. This reflects both good adherence to standards of care as well as sustainability in treatment access in Poland, as compared to other Central and Eastern European countries [29]. It needs to be emphasized that it is thanks to many stakeholders, activists, and medical experts that despite multiple medical contract re-negotiations with national healthcare agencies and scattered funding from government, the system of care and access to cART remains stable in Poland. 


\section{Action points from the meeting}

- Estimating the number of people living with HIV in Poland using mathematical modelling, but based on real data sampling.

- Reporting guidelines underlining the use of current coded identifier in surveillance and development of common identifier acceptable for all stakeholders, allowing data linkage.

- Expanding new testing options in VCT centers, i.e. rapid testing, co-testing (HIV, HCV, STI).

- Strengthening linkage to care after establishing the HIV diagnosis in non-medical settings.

- Expanding HIV testing in medical settings, starting with introducing routine HIV testing for indicator conditions as a standard procedure across different medical specialties.

- Knowing the number of HIV-positive persons already in care and on effective cART.

- Continuing to fight stigma and discrimination through the means of social education and counselling.

\section{Conflict of interest}

The authors declare no potential conflicts of interest with respect to the research, authorship, and/or publication of this article. 